Article

\title{
A parallel tracking of salivary and gut microbiota profiles can reveal maturation and interplay of early life microbial commu- nities in healthy infants
}

\author{
Sofia Reddel 1; Giuseppe Rubens Pascucci 2; Silvia Foligno 3; Federica Del Chierico 1; Pamela Vernocchi 1; \\ Alessandra Marzullo 3; Maria Grazia Pattumelli 4, Paolo Palma 2; Guglielmo Salvatori 3, \$; Lorenza Putignani 5, \$* \\ 1 Multimodal Laboratory Medicine Research Area, Unit of Human Microbiome, Bambino Gesù Children's \\ Hospital, IRCCS, Rome, Italy \\ 2 Academic Department of Pediatrics (DPUO), Research Unit of Clinical Immunology and Vaccinology, \\ Bambino Gesù Children's Hospital, IRCCS, Rome, Italy; Chair of Pediatrics, Department of Systems \\ Medicine, University of Rome "Tor Vergata", Rome, Italy \\ 3 Neonatology Department, Bambino Gesù Children's Hospital, IRCCS, Rome, Italy \\ 4 Unit of Pediatric and Neonatal Intensive Care Unit, Belcolle Hospital, Viterbo, Italy \\ 5 Department of Diagnostic and Laboratory Medicine, Unit of microbiology and diagnostic immunology, \\ Unit of Microbiomics and Multimodal Laboratory Medicine Research Area, Unit of Human Microbiome, \\ Bambino Gesù Children's Hospital, IRCCS, Rome, Italy \\ $\$$ Co-last authorship \\ * Correspondence: guglielmo.salvatori@opbg.net (G.S.); lorenza.putignani@opbg.net (L.P.)
}

\begin{abstract}
The onset and progression of the salivary and gut microbiota, the transmission and the impact of the salivary microbiota on the development of early fecal microbial communities were herein explored.We characterized the microbiota of 82 faecal and 80 salivary samples, collected from 82 healthy newborns at birth, 7, 15, 30, 90 and 180 days of life, by $16 \mathrm{~S}$ rRNA targeted-metagenomics approach. Correlation heat-maps and co-occurrence networks were used to investigate microbial taxa relationship in saliva, gut and between the two ecosystems. In saliva microbiota, Streptococcus and Staphylococcus appeared as early commensals, dominating this ecosystem through the time, while Fusobacterium, Prevotella, Porphyromonas, Granulicatella and Veillonella were late colonizers. anaerobes as Enterobacteriace, Staphylococcus and Streptococcus, were gut microbiota pioneers, followed by the anaerobic Bifidobacterium, Veillonella, Eggerthella and Bacteroides. Streptococcus, Staphylococcus and Veillonella were shared by gut and saliva ecosystems (core microbiota). Early saliva and gut microbiota seem to evolve independently driven by local adaptation strategies, with the only exception for the oral Streptococcus and Veillonella genera, involved in gut microbiota development as seeding species. A more comprehensive knowledge of how oral microbiota may affect pathophysiological conditions of gut microbiota may open new avenues on the design of postbiotics.
\end{abstract}

Keywords: oral microbiota; gut microbiota; networks analysis; ecological niche; newborns

\section{Introduction}

It is now largely recognized that microbial colonization in the human intestine begins immediately at birth [1-4] or, even before delivery, during intrauterine life [5-7]. The infant's gut microbiota is a highly dynamic community that is progressively and continuously modulated during life, with delivery and feeding representing the most relevant factors driving its onset and shaping $[1,8]$.

Alterations of gut microbiota, intended as dysbiosis, during neonatal life and infancy have been associated with paediatric disorders and with the establishment of several diseases during adult life [9,10]. Consequently, understanding gut microbiota colonization dynamics in early life is not only intriguing in terms of microbial ecology, 
but also it may give novel insights onto the relationship between microbiome treats and disease triggering [11,12].

Contrary to the enormous number of studies available on the development of infants' gut microbiota [4,13-16], information on the progressive makeup of infant oral microbiota is still limited. Only recently, a longitudinal study for the characterization of oral microbiota in infants followed since 3 months to 7 years of age was conducted [17]. Oral microbiota compositional patterns changed through the first 2 age of life, starting with "early colonizers", including Streptococcus, Veillonella and Lactobacillus spp., and ending with "late colonizers", such as Actinomyces, Porphyromonas, Abiotrophia and Neisseria [17].

Moreover, longitudinal studies focused on the simultaneous analysis of oral and intestinal microbiota development with culture-independent next-generation sequencing methodologies, are very scarce. Recently, a first study to assess microbiota differentiation across multiple body sites in newborns was conducted. However, this study considered a very small population of only six preterm infants with low birth weight [18]. Furthermore, two recent studies explored the influence of different maternal microbiota on the development of newborn gut and oral microbiota $[19,20]$.

To our knowledge, the impact of oral and gut shared bacteria was reported only in the study of Ferretti et al., but the description was limited only to taxa description without any network and correlation implication between communities [20]. In another study by Li et al. a co-occurrence network approach was exploited but in a gnotobiotic mouse model to understand the ecological invasion of salivary bacteria into the mouse gut [21].

In the herein study we addressed the establishment and development of the salivary and gut microbiota in the first six months of life in healthy breastfed newborns. Moreover, by correlation analysis and co-occurrence network approach, the ecological progression of the two ecosystems during a time course was investigated to highlight the impact, through transmission, of the salivary microbiota on the development of early fecal microbial communities.

\section{Materials and Methods}

\subsection{Study subjects and sample collection}

Eighty-two newborns born at the Unit of Gynecology and Obstetrics of Fatebenefratelli Hospital of Rome in Italy, were enrolled in this observational crosssectional study during routinely visits at Bambino Gesù Children's Hospital and Research Institute of Rome.

Inclusion criteria regarded healthy newborns vaginally delivered at term (37-42 gestational weeks) with a normal birth weight $(2.5-4.5 \mathrm{~kg})$, breastfed and with an age range of 0-180 days. The exclusion criteria included caesarean section delivery, antibiotic intake and acute or chronic gastrointestinal (GI) diseases registered in the thirty days before the starting point of both stool and saliva collection and also during the entire time course.

The Hospital Ethics Committees approved the study ("Protocol 784_OPBG_2014"). After receiving written informed consent from parents, saliva samples were collected by gently swabbing the infants' cheeks with a sterile cotton swab at 7, 15, 30, 90 and 180 days of time life. Stools were collected at birth (meconium) and subsequently from infant diapers (Table 1).

Table 1. General characteristics of neonate cohort.

\begin{tabular}{ccccccc}
\hline Characteristics & \multicolumn{7}{c}{ Age (days) } \\
\hline & 0 & 7 & 15 & 30 & 90 & 180 \\
\hline Subjects $\mathbf{( n}^{\circ}$ ) & 5 & 19 & 15 & 18 & 15 & 15 \\
\hline Saliva samples & 0 & 19 & 15 & 18 & 15 & 15 \\
\hline Stool samples & 5 & 17 & 14 & 15 & 14 & 15 \\
\hline Gestational age (mean,weeks) & 39.2 & 39.4 & 39.4 & 39.3 & 39.3 & 39.3 \\
\hline Female/male & $1 / 4$ & $9 / 10$ & $5 / 10$ & $10 / 8$ & $8 / 7$ & $9 / 6$ \\
\hline
\end{tabular}




\begin{tabular}{ccccccc}
\hline Birth weight (mean, g) & 3528 & 3418 & 3506 & 3267 & 3350 & 3221 \\
\hline $\begin{array}{c}\text { Weight at the time of stool/saliva } \\
\text { collection (mean, g) }\end{array}$ & 3430 & 3390 & 3744 & 4099 & 5828 & 6979 \\
\hline Weaning a & No & No & No & No & No & $\begin{array}{c}\text { Yes } \\
(10 / 15)\end{array}$ \\
\hline
\end{tabular}

at 180 days 10/15 infants started weaning mostly with fruit (mainly apple and pear).

All samples were collected during routinely visits at Bambino Gesù Children's Hospital and Research Institute of Rome except for meconium samples that were collected at birth at the Unit of Gynecology and Obstetrics of Fatebenefratelli Hospital, Rome, Italy.

Samples, stored at $4^{\circ} \mathrm{C}$, were then transported at controlled temperature and longterm stored at the BBMRI Biobank Microbiome node of the Human Microbiome Unit of Bambino Gesù Children Hospital until laboratory processing.

\subsection{Fecal and salivary microbiota profiles}

DNA was manually extracted from stools using QIAmp Fast DNA Stool mini kit (Qiagen, Hilden, Germany), while saliva samples were automatically extracted using the EZ1 DNA Tissue Kit and automatic extractor biorobot EZ1 following manufacturer's instructions (Qiagen, Hilden, Germany).

The variable region V3-V4 of the 16S rRNA locus was amplified using primers described in the 16S Metagenomic Sequencing Library Preparation protocol (Illumina, San Diego CA, USA). The first PCR mix was prepared with the Fast Start Hifi Taq (Roche Diagnostics, Germany) and the reaction set up as follows: initial denaturation at $95^{\circ} \mathrm{C}$ for $3 \mathrm{~min}, 32$ cycles of denaturation at $95^{\circ} \mathrm{C}$ for $30 \mathrm{~s}$, annealing at $55^{\circ} \mathrm{C}$ for $30 \mathrm{~s}$, extension at $72{ }^{\circ} \mathrm{C}$ for $30 \mathrm{~s}$, and final extension step at $72{ }^{\circ} \mathrm{C}$ for $5 \mathrm{~min}$. DNA amplicons were purified with $20 \mu \mathrm{l}$ of KAPA Pure Beads (Roche Diagnostics, Germany) and the second PCR step was performed by Nextera indexes (Illumina, USA), to obtain unique combination of barcoded sequences. The final library was purified with $50 \mu \mathrm{l}$ of KAPA Pure Beads, quantified using Quant-iT ${ }^{\mathrm{TM}}$ PicoGreen ${ }^{\circledR}$ dsDNA Assay Kit (Thermo Fisher Scientific, USA) and diluted in equimolar concentrations $(4 \mathrm{nM})$. Samples were subsequently pooled, denaturized, diluted at $7 \mathrm{pM}$ and sequenced on an Illumina MiSeqTM platform (Illumina, USA).

\subsection{Biocomputational and statistical analyses}

The Qiime v1.8 pipeline was used to analyze Illumina Miseq reads in terms of quality, length and chimera presence. Then, sequences were organized into Operational Taxonomic Units (OTUs) with a 97\% of clustering threshold of pairwise identity. PyNAST v.0.1 program was used to carry out a multiple sequence alignment against Greengenes 13_08 database with a $97 \%$ similarity for bacterial sequences [22]. The OTUs multiple sequence alignment was used to build a phylogenetic tree [23]. The microbiota biodiversity was analysed by $\alpha$ - and $\beta$-diversity performed by Phyloseq [24] of R package (version 3.6.2). To evaluate microbial richness and rarity, Shannon and Chao1 indices, respectively, were computed.

PERMANOVA test was applied on $\beta$-diversity metrics with 9999 permutations to com-pare samples at different time points. Saliva and fecal OTU tables were filtered retain OTUs present at least of $20 \%$ of all samples. Significant change in OTUs relative abundances at phylum and genus levels were assessed by Kruskal-Wallis test, corrected for multiple test ( $\mathrm{pFDR}<0.05)$. At genus level, only taxa with a relative abundance higher than 0.01 were considered for the computational analysis.

Heat-maps and co-occurrence networks were obtained from all time points included in this study. Pearson's correlation method and R package (version 3.6.2) were used to find significant correlations between relative abundance values at phylum and genus taxonomic level. Only statistically significant correlations ( $p F D R<0.05)$ were used to draw graphical representations. 
Co-occurrence networks were produced with Cytoscape 3.7.2 version and network analysis was performed with Network Analyzer plug-in.

\section{Results}

Eighty-two fecal and 80 saliva samples were collected from 82 infants through a time course described in Table 1.

\subsection{Ecological analyses of salivary and intestinal microbiota}

For saliva samples, an increase of $\alpha$-diversity was observed since the time point 15 days, which also showed the highest median value for both Shannon and Chao1 indexes. Particularly, significant differences resulted for the comparison between the time points 7 and 15 days ( $p=0.048$ and $p=0.025$ for Shannon for Chao1, respectively), while a statistically significant difference between 7 and 180 days was observed only for Shannon index ( $p=0.037$, Figure 1 ).
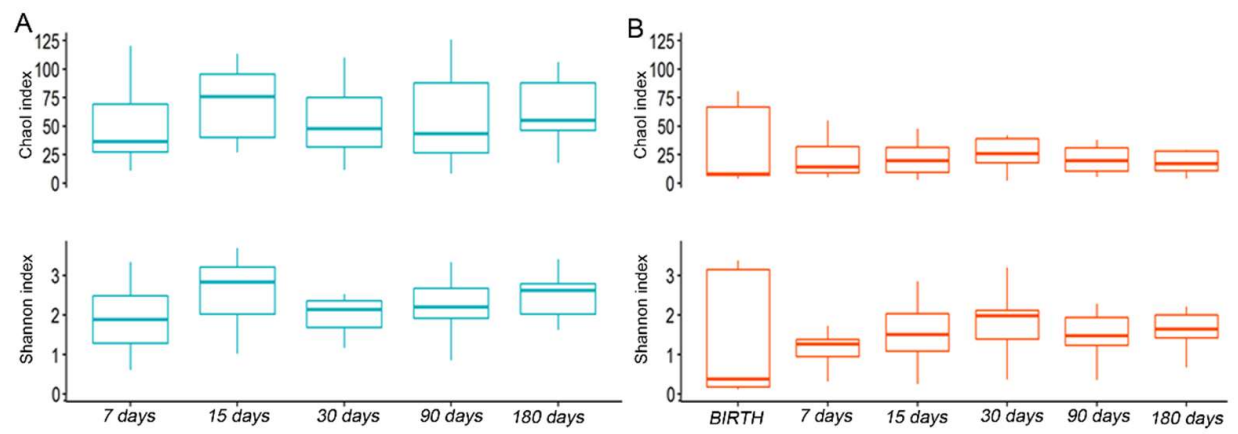

Figure 1. Box plots of $\alpha$-diversity measures by Chao1 and Shannon indexes of saliva (A) and stool (B) samples. Boxes represent the minimum and maximum values, median, $25^{\text {th }}$ and $75^{\text {th }}$ percentiles of $\alpha$-diversity indexes at the indicated time points.

The $\alpha$-diversity measures in fecal samples followed an increasing tendency during the 7-180 days' time course similar to saliva samples even if with a lower complexity (Figure 1). The highest mean value both for Shannon and Chao1 indexes was observed at 30 days. A significant difference was measured in Shannon index between 7 and 30 days $(\mathrm{p}=0.031)$ and 7 and 180 days $(\mathrm{p}=0.019)$ time points, respectively.

However, $\alpha$-diversity was significantly higher in saliva compared to stool samples at each newborns' time point, except for of values measured at 30 days (Figure 1).

Compositional dissimilarity between time-points in terms of phylogenetic relatedness was evaluated by $\beta$-diversity using the unweighted UniFrac algorithm. For both saliva and fecal samples, the OTUs distribution differences at the different timepoints, resulted statistically significant as assessed by PERMANOVA test ( $\mathrm{p}=0.001$ and $\mathrm{p}=0.032$, respectively). The separation in the first axis was stronger for fecal than saliva samples (Figure 2).

The microbiota composition across saliva and feces body matrices showed a significant different structure, as assessed by PCoA built by Unweighted algorithm with PERMANOVA analysis ( $\mathrm{p}=0.0001$; Figure 2 ). 

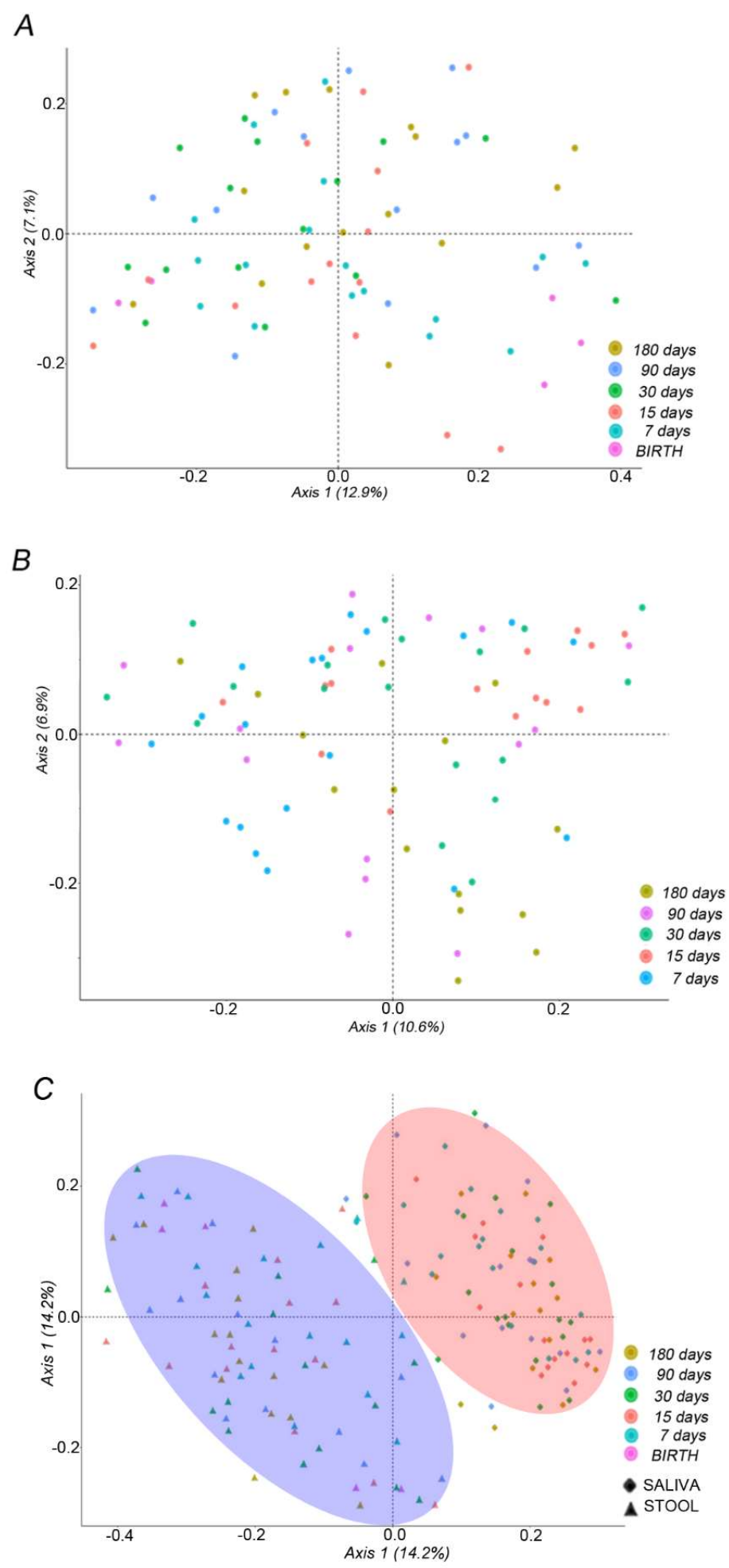

Figure 2. Principal Coordinates Analysis (PCoA) plots of $\beta$-diversity matrix of all time-points for stool (A), saliva (B) and both saliva and stool (C) samples. The shape of the symbols refers to the microbiota matrix while the color indicates the time point. Red and blue ellipses highlight saliva and stool samples, respectively. The plots show the first two principal axes for PCoA using unweighted UniFrac algorithm. 


\subsection{Salivary microbiota profiling}

The salivary microbiota was largely dominated by Firmicutes during all the time course. At 90 days there was a strong increase of Actinobacteria, but this results changed at 180 days, for most of the samples, towards an increase of Proteobacteria $(p \mathrm{FDR}<0.05)$, Fusobacteria $(p \mathrm{FDR}<0.05)$ and Bacteroidetes (Supplementary Figure 1).

At genus level, statistically significant differences were found during the time course for the following OTUs: Haemophilus (Proteobacteria), Porphyromonas and Prevotella (Bacteroidetes), Fusobacterium (Fusobacteria) and Granulicatella (Firmicutes) (Figure 3).
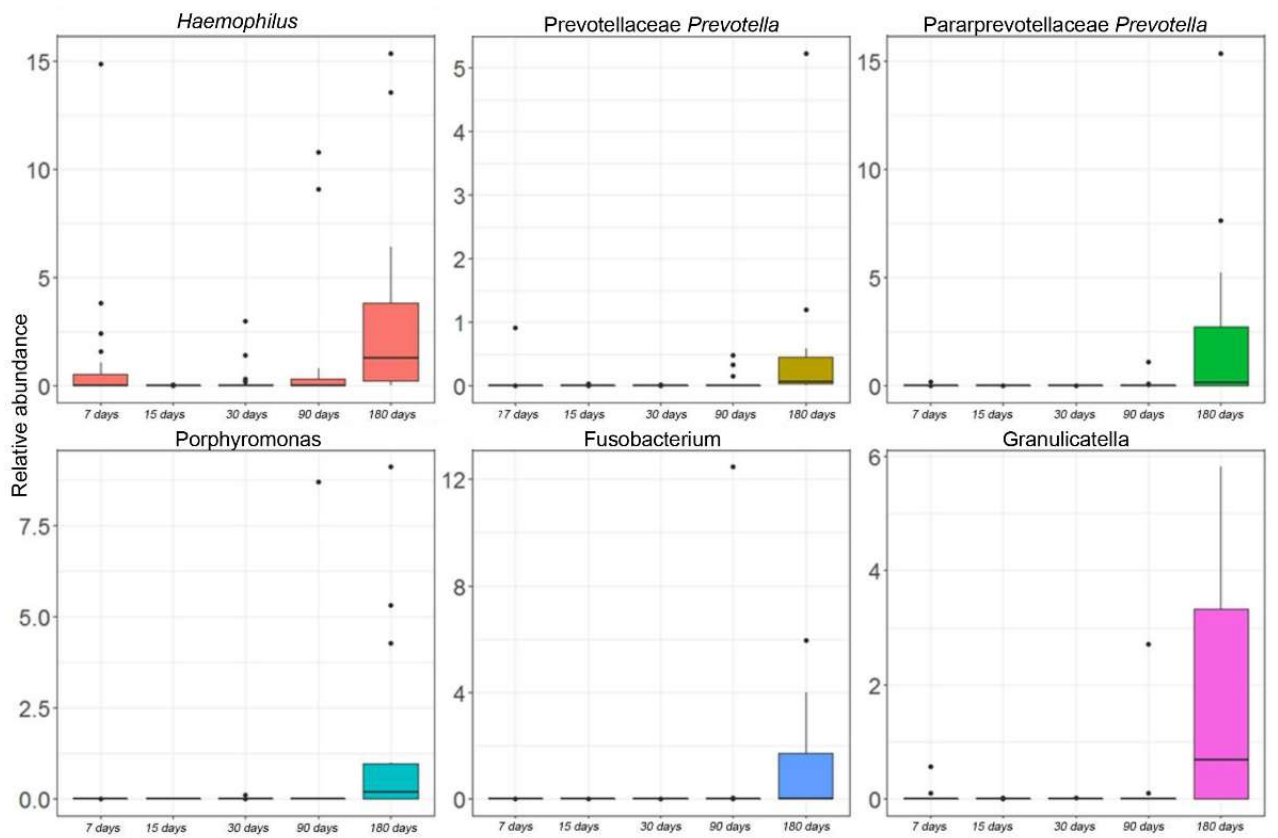

Figure 3. Kruskal-Wallis test-based OTU distribution of the salivary microbiota. The bar graphs represent the average distribution of OTUs at genus level in salivary microbiota during the first 6 months of infant's age. Only statistically significant OTUs with $\mathrm{pFDR}<0.05$ are shown.

All of them were characterized by a deep increase in their abundances at the last point of the time course.

From an analysis of OTUs shared between all-time points, we were able to identify a temporal core microbiota in saliva samples (Supplementary Table 1). The most prevalent OTUs ( $\geq 1 \%$ abundance) were Rothia, Staphylococcus, Streptococcus, Gemellaceae_g and Veillonella. Particularly, Staphylococcus and Streptococcus were the predominant genera of the infant salivary core microbiota during the first six months of age (Supplementary Table 1). In particular, Streptococcus presented a remarkable decrease of its relative abundance from $75.8 \%$ to $66.9 \%$ at 180 days' time point (Supplementary Table 1 ).

\subsection{Gut microbiota profiling}

Fecal microbiota was characterized by a more homogenous distribution of phyla compared to the salivary one. The entire time course showed a high percentage of Proteobacteria with a relative abundance distribution that varies significantly over time $(\mathrm{pFDR}<0.05)$. At day 15 there was a strong increase of Bacteroidetes with a gradually transition towards an increase of Actinobacteria until the last time point. Interestingly the phyla Fusobacteria and OD1 showed a decreasing trend from the birth over time (pFDR< 0.05) (Supplementary Figure 2).

At genus level, Kruskal-Wallis test revealed significant differences for 10 OTUs (Figure 4). In particular, Actinomyces, Bifidobacterium and Enterococcus followed an increasing trend during the entire time-course; particularly Bifidobacterium started to appear at day 7 and it significantly increased over time blowing up at day 90. Eggerthella 
and Lactobacillus abundances increased until day 90 but started to decrease from day 180; Bacillus and Staphylococcus showed a peak of abundance a day 7 but decreased during the time course. Streptococcus and Gemella showed a constant increase of their relative abundances starting since the day 15. Interestingly, Alistipes had high levels of abundance at birth but during the next time-points its levels were nearly zero (Figure 4).
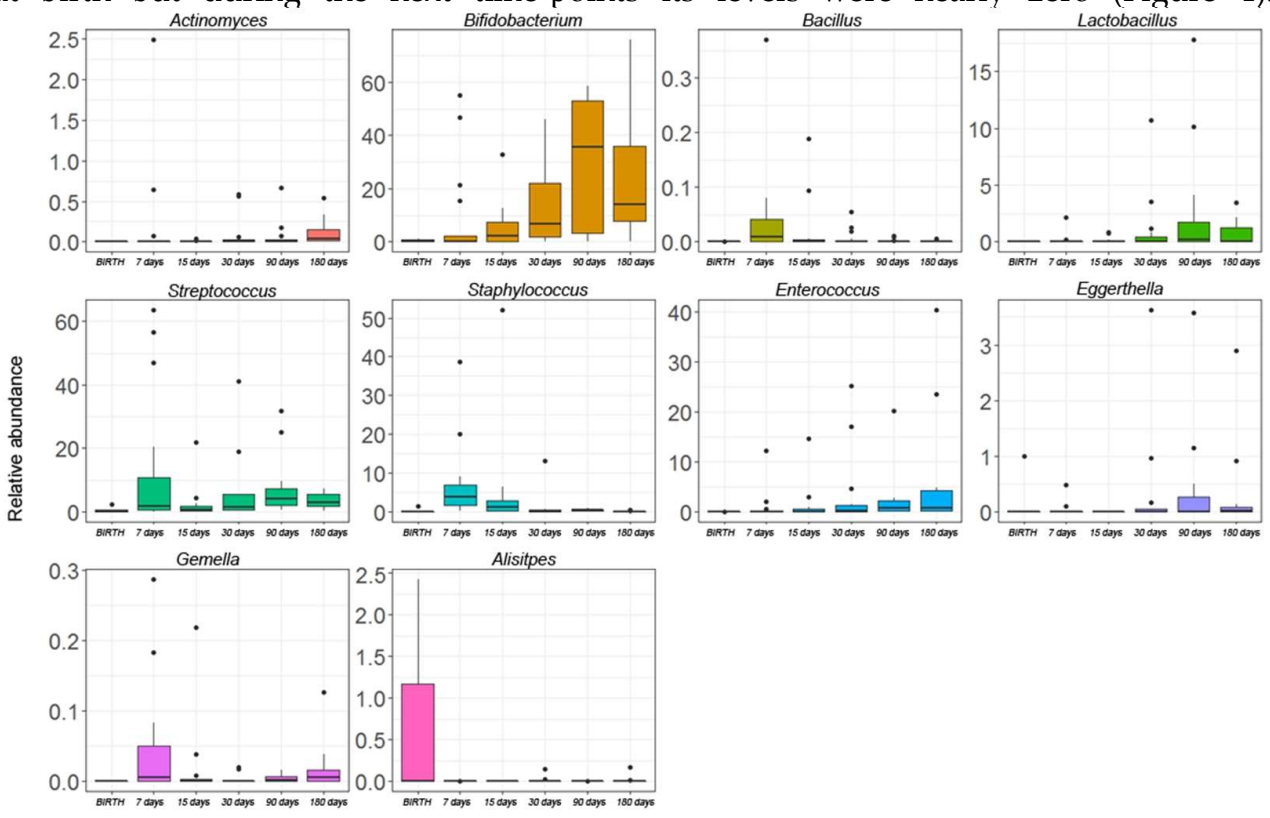

Figure 4. Kruskal-Wallis test-based OTU distribution of the gut microbiota. The bar graphs represent the average distribution of OTUs at genus level in gut microbiota during the first 6 months of infant's age. Only statistically significant OTUs with pFDR $<0.05$ are shown.

An analysis of OTUs shared from birth to the first six months of age of our infant cohort revealed the presence of a gut core microbiota composed of 14 OTUs (Supplementary Table 2). Particularly, Enterobacteriaceae_g predominated in meconium samples ( $>90 \%$ of abundance) but decreased at day 7 leaving space to other genera, including the most abundant Bifidobacterium, Bacteroides, Staphylococcus, Streptococcus and Veillonella as represented in Supplementary Table 2.

\subsection{OTUs at genus level shared between gut and salivary microbiota}

To explore the possible bacteria transit from saliva to gut ecosystem, we evaluated which OTUs were shared between fecal and salivary microbiota during the time course (Supplementary Table 3).

A deep analysis of shared OTUs between the two ecosystems revealed that at each time points an average number of 7 OTUs were present (Supplementary Table 3). In particular, Staphylococcus, Streptococcus and Veillonella were shared during all points of the time course (Supplementary Table 3).

The abundances' trend over time of Staphylococcus, Streptococcus and Veillonella was then analysed. Staphylococcus and Streptococcus were dominant and persistent colonizers in saliva samples, while in fecal samples their showed a lower abundance. Particularly, Staphylococcus had higher levels during the first days of life and decreased during time, while Streptococcus showed a different trend in both saliva and gut microbiota. In saliva, Streptoccoccus showed a decreasing trend during the last points of the time course, while in the gut microbiota a constant increase was exhibited after the day 15 (Supplementary Figure 3). Veillonella increased over time both in salivary and gut microbiota, showing higher levels in saliva samples (Supplementary Figure 3).

\subsection{OTUs correlations and network analysis at genus level in the salivary microbiota}


During evolution of the salivary microbiota, we observed an increase in the number of total OTUs correlations. Particularly, this increase is more evident in the transition from day 15 to 30 while after the day 90 we observed that correlation networks around OTUs belonging to Firmicutes, Bacteroidetes and Proteobacteria phyla started to converge into tightly condensed agglomerates (Figure 4 5).

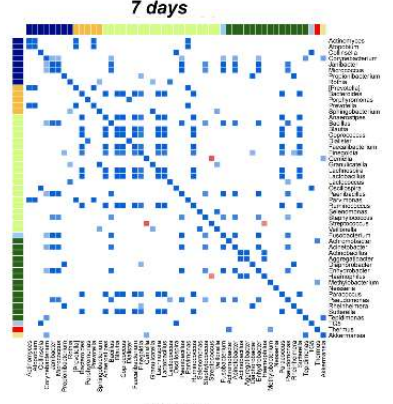

90 days

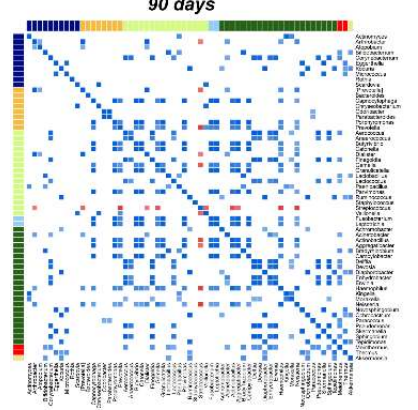

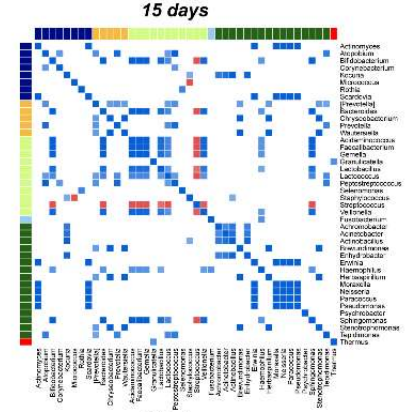

180 days

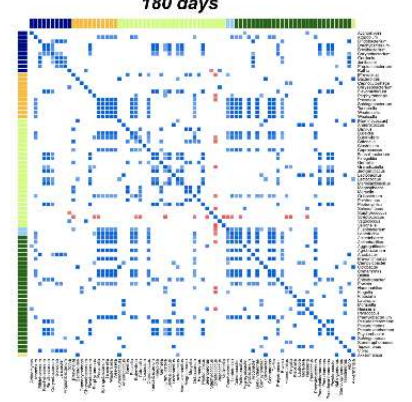

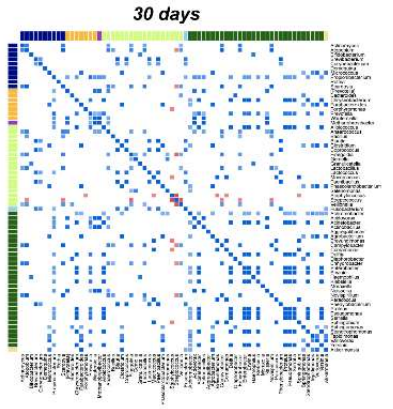

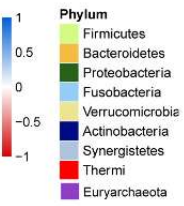

Figure 5. Pearson's correlation heat maps of salivary OTUs. Panels labeled as 7, 15, 30, 90, 180 days show the correlation (colored squares) heat maps between OTUs. Correlations are calculated by Pearson's test, only those with $\mathrm{pFDR}<0.05$ are represented. The color scale represents the correlation level: red, negative correlation values; blue, positive correlation values. OTUs are colored according to the phylum level taxonomy.

Focusing on the most abundant OTUs in the salivary microbiota, we noted that Streptococcus established only negative correlations and their number increased during the time course (Figure 5 and Figure 6, panels A-E). 

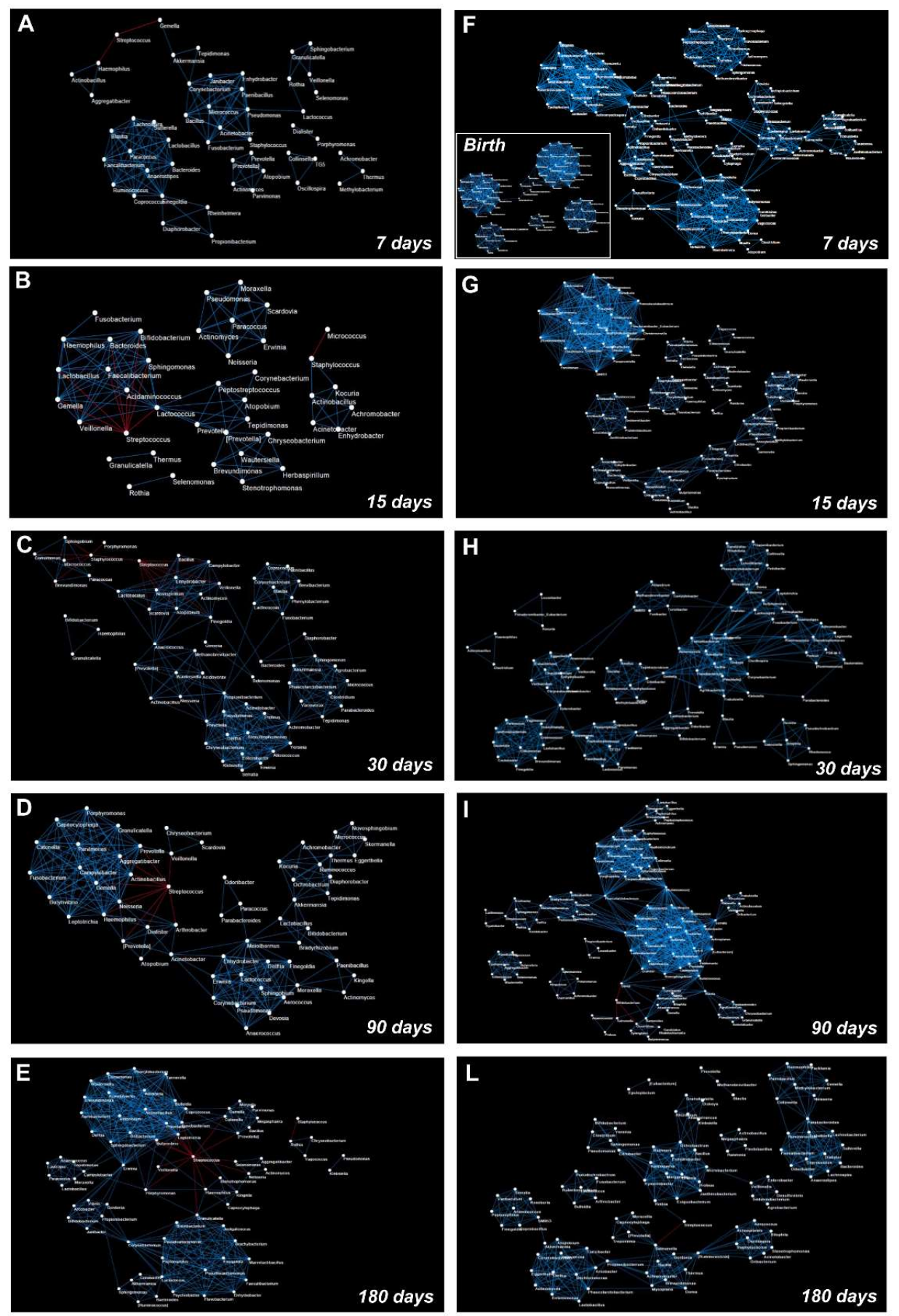

Figure 6. Graphical representation of OTUs co-occurrence networks. Panels represent OTUs cooccurrence networks of saliva and stool samples analysed at days 7, 15, 30, 90 and 180 following birth, respectively. In particular, panels from A to E represent the OTUs co-occurrence networks for saliva samples. The panels from $\mathrm{F}$ to $\mathrm{L}$ represent co-occurrence network obtained from stool samples at the same sampling time. The co-occurrence network obtained from meconium samples is represented as an inset within panel F. Blue line indicates a positive correlation and a red line indicates a negative correlation. Pearson's test was used to evaluate the correlation amongst OTUs (statistical significance was assessed with pFDR <0.05). 
Indeed, at day 7 Streptococcus negatively correlated only with Haemophylus and Gemella, the third most abundant taxa at day 7, while at day 15 it established more negative correlations, particularly with Veillonella, Gemella, Lactobacillus, Faecalibacterium, Bacteroides, Lactococcus, Bifidobacterium, Sphingomonas and Acidaminococcus (Figure 5 and Figure 6, panel B). At day 30 Streptococcus changed its negative correlations except for that with Veillonella and established new correlations with Staphylococcus, Campylobacter, Enhydrobacter, Scardovia, Novispirillum, Atopobium and Finegoldia (Figure 5 and Figure 6, panel C). At day 90, Streptococcus still negatively correlated with Veillonella and also established a negative correlation with Gemella, Haemophilus, Neisseria, Actinobacillus, Aggregatibacter, Prevotella and Dialister (Figure 5 and Figure 6, panel D). At day 180, Streptococcus negatively correlated with Veillonella and with all genera that significantly increased during the entire time course, as Haemophilus, Granulicatella, Prevotella, Porphyromonas and Fusobacterium (Figures 4-5 and Figure 6, panel E). Network analysis showed that the highest number of OTUs and their relative positive correlations condensed into two large and four small clusters at day 180 (Figure 6, panel A-E). These groups seem to evolve independently each other but some of their members such as Haemophilus, Granulicatella, Porphyromonas and Fusobacterium established an interconnection thought a negative correlation with Streptococcus (Figure 6, panel E).

The second most abundant OTU, that is Staphylococcus, showed negative correlations only at day 30 with Streptococcus, Porphyromonas, Sphingobium, Brevundimonas, Comamonas and Macrococcus. Starting from day 90 its correlations decreased and it maintained a negative correlation only with Rothia (Figure 5 and Figure 6 panels C-E).

Regarding Gemella and Veillonella, the other two most abundant OTUs in the saliva microbiota, they presented several positive correlations during the entire time course; none of these established stable relationships over time, with the only exception of a strong negative correlation with Streptococcus as stated above (Figure 5 and Figure 6, panel A-E). Rothia, the less abundant OTU amongst the five top ranking taxa, presented no significant correlations at the point of its maximum abundance (day 90), showing a negative correlation with Staphylococcus during the last time points of the study (Supplementary Table 1, Figure 5 and Figure 6, panels D-E).

\subsection{OTUs correlations and network analysis at genus level in the gut microbiota}

Based on the heat map correlation analysis we observed that the number of total correlations was higher compared to those established in the salivary microbiota (Figure 7).
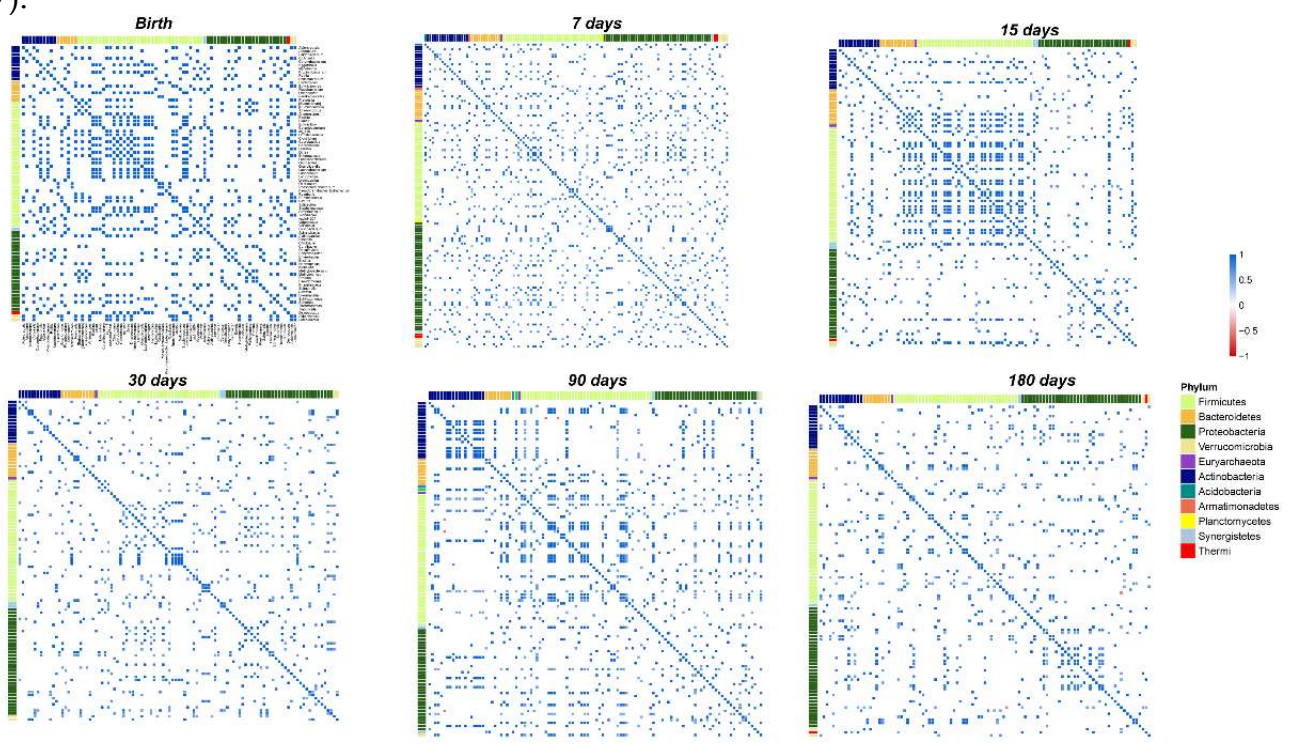

Figure 7. Pearson's correlation heat maps of faecal OTUs. Panels labelled as Birth, days 7, 15, 30, 90, 180 show the correlation (coloured squares) heat maps between OTUs. Correlations are calculated 
by Pearson's test, only those with $\mathrm{pFDR}<0.05$ are represented. The colour scale represents the correlation level of each variable: red, negative correlation values; blue, positive correlation values. OTUs are coloured according to the phylum level taxonomy.

Moreover, network analysis revealed a higher clusterization coefficient in the gut microbiota compared to the salivary microbiota (Supplementary Table 4).

Particularly, from birth to day 7 of life there was a strong increase in the number of total correlations (Figure 6, panel F and Figure 7).

Furthermore, only positive correlations between OTUs were detected at days 7, 15 and 30 (Figure 6, panels F-H and Figure 7).

Going on the specific correlations analysis, we focused on the correlations established by the OTUs that we speculated were transferred from saliva to stool and on the correlations by the 10 OTUs which significantly changed during the time course of the gut microbiota maturation (Figure 4 and Supplementary Table 2).

At day 7, we observed that Streptococcus established a positive correlation with Prevotella, Bacillus, Enterococcus, Haemophylus, Coprococcus, Dorea, Faecalibacterium and Lactococcus. At day 15, there was a high number of positive correlations established by OTUs belonging to Firmicutes. Particularly, Bifidobacterium positively correlated with Staphylococcus, Bacillus, Haemophylus and Gemella. Another positive correlation was observed between Bacillus and Streptococcus (Figure 6, panel G and Figure 7).

At day 30, Bifidobacterium changed its positive correlations and interacted with Actinomyces that started to increase since this time point on (Figure 4, Figure 6, panel $\mathrm{H}$ and Figure 7). Moreover, Actinomyces was part of an interaction network that involved Lactobacillus and Enterococcus, which were significantly increased at this time point and that was in turn connected with another network of positive correlations in which Eggerthella and Veillonella were present (Figure 6, panel H). Another triangular cluster of positive interaction involved Streptococcus, Bacillus and Staphylococcus, which were connected with a positive correlation with Gemella (Figure 6, panel H and Figure 7).

At day 90, negative correlations were detected for Bifidobacterium and Dorea, Clostridium and Salmonella. Moreover, Bacillus positively correlated with a cluster of nodes in which Actinomyces, Eggerthella and Lactobacillus were present. Moreover, Bacillus showed a positive correlation with Staphylococcus (Figure 6, panel I and Figure 7). Three OTUs, i.e., Veillonella, Streptococcus and Enterococcus, formed a small independent cluster of nodes (Figure 6, panel I and Figure 7). At day 180, Actinomyces, Eggerthella and Lactobacillus continued to be connected by positive correlations and positively cooperated with Enterococcus within the same cluster of nodes (Figure 6, panel I and Figure 7). Furthermore, Streptococcus established a negative correlation with Salmonella.

\subsection{OTUs correlations and co-occurrence network analysis at genus level between salivary and} gut microbiota

To study the relationship between microbial communities colonizing human saliva and gut, co-occurrence network was used (Figure 8). For this analysis we focused on the OTUs shared between salivary and gut microbiota (Supplementary Table 3).

Particularly, at day 7 we observed positive correlations between: Staphylococcus (saliva) versus Staphylococcus (stool), Rothia (saliva) versus Rothia (stool), Veillonella (saliva) versus Veillonella (stool) and Gemella (saliva) versus Gemella (stool) (Figure 8, panel A). Interestingly, from network analysis we visualized a cluster of interactions established by Staphylococcus (saliva) with several members of Actinobacteria, Proteobacteria and Firmicutes phyla. Particularly, Staphylococcus negatively correlated with Streptococcus, Bacillus and Acinetobacter and positively correlated with Pseudomonas and Micrococcus, all of them in stools (stool). 


\section{A}

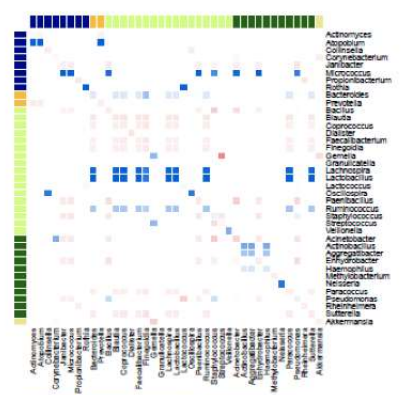

B

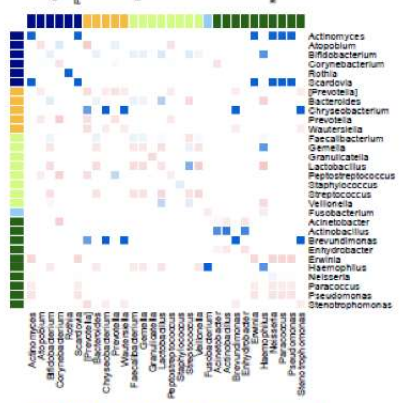

C

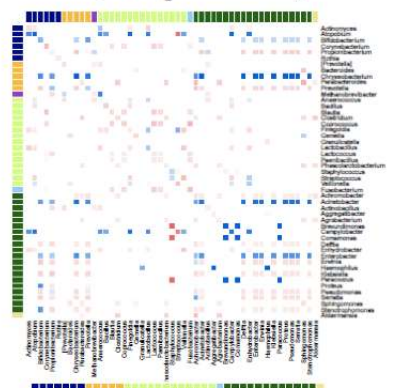

D

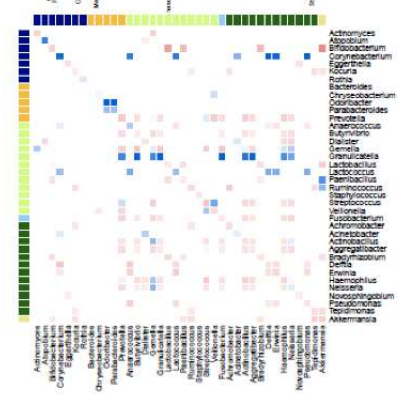

E

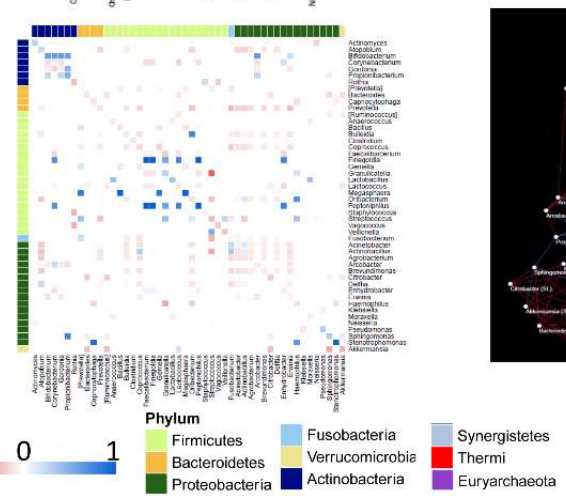

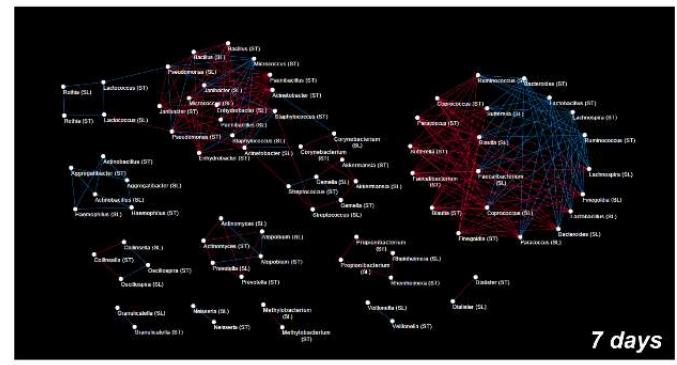
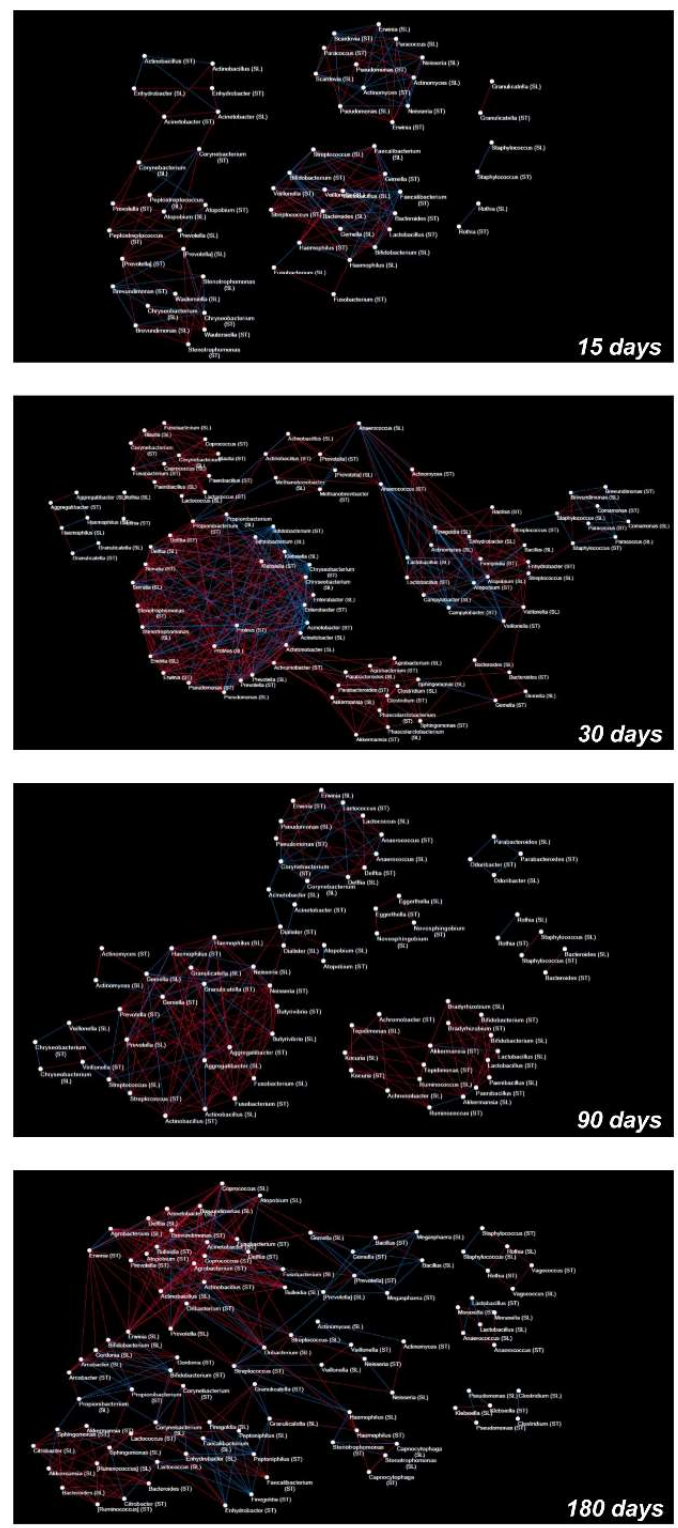

Figure 8. Correlation heat maps and OTUs co-occurrence networks of saliva versus stool microbiota from 7 to 180 days following birth. On the left side, panels A-E show the correlation (coloured squares) heat maps between saliva versus stool OTUs at days 7 (A), 15 (B), 30 (C), 90 (D), 180 (E). Correlations are calculated by Pearson's test and only those with a significance $\mathrm{pFDR}<0.05$ are represented. The two coloured scale within panel A indicates the heat maps correlation values and 
the phylum-related OTU, respectively. On the right side of panels A-E are represented OTUs cooccurrence networks calculated based on heat map correlation data. Blue line indicates a positive correlation and a red line indicates a negative correlation.

Moreover, Streptococcus (saliva) and Streptococcus (stool) presented a weak negative correlation, however the Streptococcus (saliva) generated a negative quadrangular correlation with Gemella (saliva), Gemella (stool) and Streptococcus (stool) (Figure 8, panel A).

As showed in Figure 8, panel A, a dense hub of positive and negative interactions was established between OTUs belonging to Proteobacteria and Firmicutes characterized in both ecosystems. At 15 days, an isolated weak positive correlation between Staphylococcus (saliva) and Staphylococcus (stool) occurred while correlations between Streptococcus (saliva vs. stool) and Gemella (saliva vs. stool) changed into negative (Figure 8, panel B). Network analysis showed that Streptococcus, Gemella and Veillonella characterized in stool and saliva samples were part of a dense cluster of nodes (Figure 8, panel B).

At day 30 Staphylococcus (saliva) still positively correlated with Staphylococcus and Streptococcus (stool) but established strong negative correlations with low abundant stool OTUs as Paracoccus, Comamonas, and Brevundimonas. At this time point, Streptococcus (saliva) did not correlate with Streptococcus (stool), but presented strong negative correlations with Atopobium (stool), Campylobacter (stool) and Veillonella (stool) (Figure 8, panel C). From network analysis, a dense hub of positive and negative correlations involved several OTUs characterized in both saliva and gut microbiota, the great majority belonging to Proteobacteria phylum (Figure 8, panel C).

At day 90, a negative correlation for each taxa, Staphylococcus, Streptococcus and Veillonella characterized in saliva and stool, respectively. Moreover, Streptococcus and Veillonella found in saliva microbiota influenced with positive correlations Veillonella and Streptococcus belonging to gut microbiota. Strong positive correlations were found between Granulicatella and Corynebacterium (saliva) with Granulicatella and Corynebacterium (stool), respectively.

The positive correlation between Gemella (saliva) and Gemella (stool) was weak and probably due to its increase in the stool and to a parallel decrease in the salivary microbiota. Moreover, Gemella (saliva) positively interacted with Granulicatella (stool) (Figure 8, panel D). The corresponding co-occurrence network showed that Streptococcus, Veillonella, Gemella, Haemophylus and several OTUs belonging to Entrobacteriaceae present in stool and saliva interacted each other within a dense cluster of positive and negative correlations (Figure 8, panel D and Supplementary Table 3).

At day 180, positive correlations between Granulicatella and Staphylococcus (saliva versus stool) and negative correlations between Streptococcus, Veillonella and Haemophilus (saliva versus stool) were observed. Furthermore, Streptococcus and Veillonella found in saliva continued to present positive correlations with Veillonella and Streptococcus observed in the gut microbiota, respectively.

\section{Discussion}

Microbiota colonization dynamics in infancy is a topic of huge interest in the field of microbial ecology and human health [25,26]. In this scenario, very little is known about the development of the infant oral microbial ecosystem. Interestingly, we found a core oral microbiota formed mainly by Streptococcus and Staphylococcus (approximately 90\% of the total oral microbiota) and, unlike the gut microbiota dynamics, was more stable during the first six months of infants' life. In agreement with other studies, Streptococcus appeared to rapidly dominate oral microbiota ecosystem and to persist during all the time course, with a relative abundance that spanned from $67 \%$ to $79 \%[17,21,27-29]$. During this period, as assessed by correlation heat maps and network co-occurrence analyses, Streptococcus intertwined a dense web of only negative correlations with other oral bacteria indicating its competition with other species for colonization purposes. Streptococcus has been 
demonstrated to be one of the prevalent bacterial genus of the breast milk ecosystem [3033] and particularly Streptococcus salivarius has been frequently found in the oral cavity of breastfed infants and associated to the first oligosaccharide stimuli [34]. Furthermore, breast milk creates an immunoglobulin A1 (IgA1)-rich ecological niche in the new-born oral cavity allowing a rapid colonization of Streptococcus spp. capable to cleavage IgA1 [35]. Moreover, this microorganism has the ability to adhere and colonize the oral mucosal surface lining [36].

A second dominant OTU observed in the salivary microbiota was Staphylococcus, previously found to be part of milk ecosystem [27] and thus, similarly to Streptococcus, possibly acquired through breastfeeding from milk or from mother skin [37,38]. Moreover, both Streptococcus and Staphylococcus are oxygen tolerant [39] and this may explain their presence as early commensals in the salivary microbiota. The production and excretion of metabolic products of these pioneers promote a change of the oral environment that we observed during our time course, thus favouring the growth and selection of other bacteria, including more strictly anaerobes [40], as proposed for gut microbiota [41]. In particular, Veillonella showed an increase of its relative abundance at 180 days' time point together with Haemophylus, Porphyromonas, Prevotella, Fusobacterium and Granulicatella, indicating that only at this stage the environment is suitable for their establishment in the oral cavity. In this new niche, Streptococcus relative abundance dropped from $75.8 \%$ to $67 \%$. Its ability to inhibit the growth of other bacterial species underwent a turnaround behaviour since an increase of oral Veillonella, Haemophylus, Porphyromonas, Prevotella, Fusobacterium and Granulicatella was found. The more reduced oral environment could explain the reason why Veillonella, which is continuous transferred from human milk to neonate's oral cavity during breastfeeding, starts to colonize infants' mouth only after 6 six months of age [42,43]. Regarding Haemophylus, Porphyromonas, Prevotella, Fusobacterium and Granulicatella, they were all linked to the development of a more mature oral microbiome $[17,21,44]$. Particularly, Granulicatella is considered a common dental plaque inhabitant, and in agreement with other studies, which followed the development of infant oral microbiota, an increase of its abundance was observed after 6 months of age [39,45]. It was hypothesized that this increment was due to teeth eruption, during 6-8 months of age, when new ecological niches are created, promoting its colonization [17].

In the herein study, analysing gut ecosystems' kinetics, we found the presence of bacterial communities also in meconium, thus corroborating the hypothesis that microbial exposure may start before delivery with early bacteria pioneers derived from the maternal microbiota $[4,5,7,10]$. Particularly, meconium ecosystem resulted characterized by the dominance of Enterobateriaceae and Bacteroides probably derived from the exposure of the new-born to mothers' microbiota during delivery [46,47]. Moreover, by an in-deep analysis of the gut microbiota composition during the entire time course a core gut microbiota was characterised. This core constituted an ecological stable niche important for the establishment of the future microbiota. The other intestinal transient colonizers, appeared at different time points and were mostly influenced by external (e.g., feeding and environment) and internal factors (e.g., gut maturation, gut oxygen levels and infants' aging) [10].

Particularly, we found the dominance of Enterobacteriaceae and Staphylococcus during the first week of life, followed by Streptococcus colonization, suggesting that the facultative anaerobes are the first colonizers in the human gut. They provide a reduced environment favourable for the establishment of later occurring anaerobic bacteria such as Bifidobacterium, Eggerthella, Bacteroides and Veillonella [46,48-50]. As assessed with oral microbiota, in the process of transition from an oxygen-tolerant to a more anaerobic gut microbiota, a possible interaction between Streptococcus spp. and Veillonella, towards the last time points of the time course, could be established. Once reached the gut, the human milk [ $7 \%$ lactose and 1\% human milk oligosaccharides (HMOs)] becomes substrate of several bacteria as Streptococcus, which is involved in human milk fermentation transforming lactose into lactate [51]. At the same time, Veillonella may be able to utilize 
the formed lactate to produce propionate, in a cross-feeding phenomenon [52-54]. The more reduced environment and the presence of HMOs induce the colonization of gut microbiota by Bifidobacterium, which is a characteristic colonizer of the human milk and is important for inhibiting the growth of pathogenic microorganisms, modulating mucosal barrier function, and promoting immunological and inflammatory responses $[27,55,56]$.

Gut microbiota heat maps and co-occurrence networks revealed that, up to 15 days, Bifidobacterium correlated positively with oral- or breastfeed-belonging bacteria such as Staphylococcus, Streptococcus, Lactobacillus, Gemella and Haemophylus. Moreover, in the following time points, when the gut ecosystem starts to become a more anaerobic, new positive and negative correlations took place with Veillonella, Bacteroides, Clostridium and several members of Proteobacteria, associated to a more mature gut microbiota [13,41]. Thus, confirming that in the development of infants' physiological microbiota, environmental conditions, like $\mathrm{pH}$, oxygen levels and nutrients availability influence microbial species selection and vice versa, in a cascade of time dependent events.

The mouth represents the main route followed by bacteria to reach the gastrointestinal tract. The influence of oral microbiota on the shape of gut microbiota is described into few studies. In adults these two communities have low overlap, while in infants the role of oral-gut axis in gut microbiota development is certainly important $[20,27,53,57]$. Unfortunately, in these studies no correlation heat maps, or co-occurrence networks were performed. Indeed, these approaches were applied to understand ecological invasion of salivary bacteria only into the gut on gnotobiotic mice [21].

In our study, ecological analyses revealed that both the composition and the structure of the two microbiota ecosystems, evolve as the infants' grow. Thus the maturation of the microbiota is a nonrandom process but it is driven by specific interactions between taxa that change during the time course of the programming [13,27,58].

Interestingly, the salivary microbiota showed an increase in total correlations from 15 to 30 days, probably due to the milk maturation. Indeed, the number of interactions among OTUs may increase proportionally with the higher intake of nutrients, linked not only to breast milk composition but also to the increased volume of the milk intake [46,54].

Our study showed that oral and gut microbiota share during the entire time course three seminal OTUs, namely Streptococcus, Veillonella and Staphylococcus, and highlighted their ability to colonize the gut microbiota in a differential way.

Regarding Staphylococcus, its relative abundance in salivary microbiota remained almost stable during all time course, while into the gut decreased over time probably due to the development of a more anaerobic environment. Correlation heat maps and network analyses of both saliva and gut ecosystems revealed that at 7 days, when Staphylococcus reached a very high concentration in oral and gut microbiota, a dense hub of connections became established with Acinetobacter, Bacillus, Paenibacillus, Pseudomonas, Janibacter, Enhydrobacter and Micrococcus in the stools. During the remaining time points, oral and gut Staphylococcus presented a very low number of connections (maximum three) with members of both gut and oral microbiota. Until the end of the time course, Staphylococcus minimally interacted with other microorganisms in stool and saliva. Thus, oral Staphylococcus seemed to not influence the shape of gut microbiota.

On the other site, Streptococcus and Veillonella established a cooperative interaction during the last points of the time course in both oral and gut ecosystems. Definitely, comparing oral and gut microbiota at 90 and 180 days' time points, the oral and gut Streptococcus and Veillonella interacted each other within the same cluster. These results support the hypothesis that, not only external and/or internal factors (i.e., environmental, feeding mode, oxygen level, age) are involved in the development of the specialized oral and gut microbiota but also some specific oral OTUs may take part into the gut microbiota modelling processes.

\section{Conclusions}


In conclusion, the findings from our small but homogeneous cohort continue to enlarge the knowledge on the progressive construction of infants' microbiota.

From an ecological point of view, our results suggest that the process of microbiota assembly is driven principally by local adaptation to the environmental niche. Moreover, we hypothesize a possible influence of some oral bacteria in the process of gut microbiota development.

However, further studies at species and strain level are needed to confirm a direct transmission; hence, shotgun sequencing approaches should be recommended to track the bacteria moving from a district to another one. The deep knowledge of how oral microbiota intervenes in the modelling of gut microbiota in pathophysiology may open new avenues on the opportunity to develop targeted postbiotic treatments.

Supplementary Materials: The following are available online at www.mdpi.com/xxx/s1, Supplementary Table 1. Average of relative abundances of OTUs shared in saliva samples (core saliva microbiota) in all points of the time-course. Supplementary Table 2. Average of elative abundances of OTUs shared in fecal samples (core gut microbiota) in all points of the time-course. Supplementary Table 3. OTUs shared between gut and salivary microbiota of the cohort of infants at each point of the time-course. Supplementary Table 4. Characteristics of networks of saliva, stool and saliva versus stool samples. Supplementary Figure 1. Beta-diversity analysis of stool and saliva samples in comparison. Supplementary Figure 2. Panel A. OTUs distribution of the salivary microbiota at phylum level. Panel B. Histograms are referred to Kruskal-Wallis test-based phyla distribution with a pFDR $<0.05$. Supplementary Figure 3. Panel A. OTUs distribution of the gut microbiota at phylum level. The bar graphs represent the average distribution of OTUs. Panel B. Histograms above are referred to Kruskal-Wallis test-based phyla distribution with a pFDR $<0.05$. Supplementary Figure 4. Colonization trends of the bacterial genera shared in all points of the time course between salivary (A) and gut (B) ecosystems.

Author Contributions: Conceptualization, L.P.; and G.S.; methodology, F.D.C., S.R. and. S.F.; formal analysis, S.R. and G.P.R.; investigation, S.R., G.P.R., S.F., F.D.C., P.V., A.M., M.G.P. and P.P.; resources, L.P. and G.S.; data curation, S.F., S.R., and F.D.C.; writing-original draft preparation, S.R. and L.P.; writing-review and editing, F.D.C., S.R. and L.P. All authors have read and agreed to the published version of the manuscript.

Funding: This work was supported by the Italian Ministry of Health (Ricerca Corrente 2016, 2017, 2018, 2019 and 5 \% 2020, assigned to LP). Also Dicofarm cofounded the study.

Data Availability Statement: All raw sequences have been archived in NCBI database: PRJNA753008 (https://www.ncbi.nlm.nih.gov/bioproject).

Conflicts of Interest: The authors declare no conflict of interest.

Acknowledge. Authors thank Dr. Andrea Quagliariello for preliminary statistical data analysis and Dr. Andrea Petrucca for critical reading of the manuscript.

\section{References}

1. Penders, J.; Thijs, C.; Vink, C.; Stelma, F.F.; Snijders, B.; Kummeling, I.; van den Brandt, P.A.; Stobberingh, E.E. Factors Influencing the Composition of the Intestinal Microbiota in Early Infancy. PEDIATRICS 2006, 118, 511521, doi:10.1542/peds.2005-2824.

2. Dominguez-Bello, M.G.; Costello, E.K.; Contreras, M.; Magris, M.; Hidalgo, G.; Fierer, N.; Knight, R. Delivery Mode Shapes the Acquisition and Structure of the Initial Microbiota across Multiple Body Habitats in Newborns. Proc. Natl. Acad. Sci. 2010, 107, 11971-11975, doi:10.1073/pnas.1002601107.

3. Bokulich, N.A.; Chung, J.; Battaglia, T.; Henderson, N.; Jay, M.; Li, H.; D Lieber, A.; Wu, F.; Perez-Perez, G.I.; Chen, Y.; et al. Antibiotics, Birth Mode, and Diet Shape Microbiome Maturation during Early Life. Sci. Transl. Med. 2016, 8, 343ra82, doi:10.1126/scitranslmed.aad7121.

4. Nagpal, R.; Tsuji, H.; Takahashi, T.; Nomoto, K.; Kawashima, K.; Nagata, S.; Yamashiro, Y. Ontogenesis of the Gut Microbiota Composition in Healthy, Full-Term, Vaginally Born and Breast-Fed Infants over the First 3 Years of Life: A Quantitative Bird's-Eye View. Front. Microbiol. 2017, 8, 1388, doi:10.3389/fmicb.2017.01388.

5. Aagaard, K.; Ma, J.; Antony, K.M.; Ganu, R.; Petrosino, J.; Versalovic, J. The Placenta Harbors a Unique Microbiome. Sci. Transl. Med. 2014, 6, 237ra65, doi:10.1126/scitranslmed.3008599. 
6. Collado, M.C.; Rautava, S.; Aakko, J.; Isolauri, E.; Salminen, S. Human Gut Colonisation May Be Initiated in Utero by Distinct Microbial Communities in the Placenta and Amniotic Fluid. Sci. Rep. 2016, 6, 23129, doi:10.1038/srep23129.

7. Chu, D.M.; Ma, J.; Prince, A.L.; Antony, K.M.; Seferovic, M.D.; Aagaard, K.M. Maturation of the Infant Microbiome Community Structure and Function across Multiple Body Sites and in Relation to Mode of Delivery. Nat. Med. 2017, 23, 314-326, doi:10.1038/nm.4272.

8. Gritz, E.C.; Bhandari, V. The Human Neonatal Gut Microbiome: A Brief Review. Front. Pediatr. 2015, 3, 17, doi:10.3389/fped.2015.00017.

9. Rautava, S.; Luoto, R.; Salminen, S.; Isolauri, E. Microbial Contact during Pregnancy, Intestinal Colonization and Human Disease. Nat. Rev. Gastroenterol. Hepatol. 2012, 9, 565-576, doi:10.1038/nrgastro.2012.144.

10. Milani, C.; Duranti, S.; Bottacini, F.; Casey, E.; Turroni, F.; Mahony, J.; Belzer, C.; Delgado Palacio, S.; Arboleya Montes, S.; Mancabelli, L.; et al. The First Microbial Colonizers of the Human Gut: Composition, Activities, and Health Implications of the Infant Gut Microbiota. Microbiol. Mol. Biol. Rev. MMBR 2017, 81, e00036-17, doi:10.1128/MMBR.00036-17.

11. Arrieta, M.-C.; Stiemsma, L.T.; Amenyogbe, N.; Brown, E.M.; Finlay, B. The Intestinal Microbiome in Early Life: Health and Disease. Front. Immunol. 2014, 5, 427, doi:10.3389/fimmu.2014.00427.

12. Honda, K.; Littman, D.R. The Microbiota in Adaptive Immune Homeostasis and Disease. Nature 2016, 535, 75-84, doi:10.1038/nature18848.

13. Bäckhed, F.; Roswall, J.; Peng, Y.; Feng, Q.; Jia, H.; Kovatcheva-Datchary, P.; Li, Y.; Xia, Y.; Xie, H.; Zhong, H.; et al. Dynamics and Stabilization of the Human Gut Microbiome during the First Year of Life. Cell Host Microbe 2015, 17, 690-703, doi:10.1016/j.chom.2015.04.004.

14. Koenig, J.E.; Spor, A.; Scalfone, N.; Fricker, A.D.; Stombaugh, J.; Knight, R.; Angenent, L.T.; Ley, R.E. Succession of Microbial Consortia in the Developing Infant Gut Microbiome. Proc. Natl. Acad. Sci. U. S. A. 2011, 108 Suppl 1, 4578-4585, doi:10.1073/pnas.1000081107.

15. Palmer, C.; Bik, E.M.; DiGiulio, D.B.; Relman, D.A.; Brown, P.O. Development of the Human Infant Intestinal Microbiota. PLoS Biol. 2007, 5, e177, doi:10.1371/journal.pbio.0050177.

16. Hill, C.J.; Lynch, D.B.; Murphy, K.; Ulaszewska, M.; Jeffery, I.B.; O’Shea, C.A.; Watkins, C.; Dempsey, E.; Mattivi, F.; Tuohy, K.; et al. Evolution of Gut Microbiota Composition from Birth to 24 Weeks in the INFANTMET Cohort. Microbiome 2017, 5, 4, doi:10.1186/s40168-016-0213-y.

17. Dzidic, M.; Collado, M.C.; Abrahamsson, T.; Artacho, A.; Stensson, M.; Jenmalm, M.C.; Mira, A. Oral Microbiome Development during Childhood: An Ecological Succession Influenced by Postnatal Factors and Associated with Tooth Decay. ISME J. 2018, 12, 2292-2306, doi:10.1038/s41396-018-0204-z.

18. Costello, E.K.; Carlisle, E.M.; Bik, E.M.; Morowitz, M.J.; Relman, D.A. Microbiome Assembly across Multiple Body Sites in Low-Birthweight Infants. mBio 2013, 4, e00782-00713, doi:10.1128/mBio.00782-13.

19. Drell, T.; Štšepetova, J.; Simm, J.; Rull, K.; Aleksejeva, A.; Antson, A.; Tillmann, V.; Metsis, M.; Sepp, E.; Salumets, A.; et al. The Influence of Different Maternal Microbial Communities on the Development of Infant Gut and Oral Microbiota. Sci. Rep. 2017, 7, 9940, doi:10.1038/s41598-017-09278-y.

20. Ferretti, P.; Pasolli, E.; Tett, A.; Asnicar, F.; Gorfer, V.; Fedi, S.; Armanini, F.; Truong, D.T.; Manara, S.; Zolfo, M.; et al. Mother-to-Infant Microbial Transmission from Different Body Sites Shapes the Developing Infant Gut Microbiome. Cell Host Microbe 2018, 24, 133-145.e5, doi:10.1016/j.chom.2018.06.005.

21. Li, B.; Ge, Y.; Cheng, L.; Zeng, B.; Yu, J.; Peng, X.; Zhao, J.; Li, W.; Ren, B.; Li, M.; et al. Oral Bacteria Colonize and Compete with Gut Microbiota in Gnotobiotic Mice. Int. J. Oral Sci. 2019, 11, 10, doi:10.1038/s41368-018-0043-9.

22. Caporaso, J.G.; Kuczynski, J.; Stombaugh, J.; Bittinger, K.; Bushman, F.D.; Costello, E.K.; Fierer, N.; Peña, A.G.; Goodrich, J.K.; Gordon, J.I.; et al. QIIME Allows Analysis of High-Throughput Community Sequencing Data. Nat. Methods 2010, 7, 335-336, doi:10.1038/nmeth.f.303.

23. DeSantis, T.Z.; Hugenholtz, P.; Larsen, N.; Rojas, M.; Brodie, E.L.; Keller, K.; Huber, T.; Dalevi, D.; Hu, P.; Andersen, G.L. Greengenes, a Chimera-Checked 16S RRNA Gene Database and Workbench Compatible with ARB. Appl. Environ. Microbiol. 2006, 72, 5069-5072, doi:10.1128/AEM.03006-05.

24. McMurdie, P.J.; Holmes, S. Phyloseq: An R Package for Reproducible Interactive Analysis and Graphics of Microbiome Census Data. PloS One 2013, 8, e61217, doi:10.1371/journal.pone.0061217.

25. Brugman, S.; Perdijk, O.; van Neerven, R.J.J.; Savelkoul, H.F.J. Mucosal Immune Development in Early Life: Setting the Stage. Arch. Immunol. Ther. Exp. (Warsz.) 2015, 63, 251-268, doi:10.1007/s00005-015-0329-y.

26. Sudo, N.; Sawamura, S.; Tanaka, K.; Aiba, Y.; Kubo, C.; Koga, Y. The Requirement of Intestinal Bacterial Flora for the Development of an IgE Production System Fully Susceptible to Oral Tolerance Induction. J. Immunol. Baltim. Md 1950 1997, 159, 1739-1745. 
27. Biagi, E.; Quercia, S.; Aceti, A.; Beghetti, I.; Rampelli, S.; Turroni, S.; Faldella, G.; Candela, M.; Brigidi, P.; Corvaglia, L. The Bacterial Ecosystem of Mother's Milk and Infant's Mouth and Gut. Front. Microbiol. 2017, 8, 1214, doi:10.3389/fmicb.2017.01214.

28. Cephas, K.D.; Kim, J.; Mathai, R.A.; Barry, K.A.; Dowd, S.E.; Meline, B.S.; Swanson, K.S. Comparative Analysis of Salivary Bacterial Microbiome Diversity in Edentulous Infants and Their Mothers or Primary Care Givers Using Pyrosequencing. PloS One 2011, 6, e23503, doi:10.1371/journal.pone.0023503.

29. Luo, A.H.; Yang, D.Q.; Xin, B.C.; Paster, B.J.; Qin, J. Microbial Profiles in Saliva from Children with and without Caries in Mixed Dentition. Oral Dis. 2012, 18, 595-601, doi:10.1111/j.1601-0825.2012.01915.x.

30. Boix-Amorós, A.; Collado, M.C.; Mira, A. Relationship between Milk Microbiota, Bacterial Load, Macronutrients, and Human Cells during Lactation. Front. Microbiol. 2016, 7, 492, doi:10.3389/fmicb.2016.00492.

31. Rodríguez, J.M. The Origin of Human Milk Bacteria: Is There a Bacterial Entero-Mammary Pathway during Late Pregnancy and Lactation? Adv. Nutr. Bethesda Md 2014, 5, 779-784, doi:10.3945/an.114.007229.

32. Jost, T.; Lacroix, C.; Braegger, C.; Chassard, C. Assessment of Bacterial Diversity in Breast Milk Using CultureDependent and Culture-Independent Approaches. Br. J. Nutr. 2013, 110, 1253-1262, doi:10.1017/S0007114513000597.

33. Pannaraj, P.S.; Li, F.; Cerini, C.; Bender, J.M.; Yang, S.; Rollie, A.; Adisetiyo, H.; Zabih, S.; Lincez, P.J.; Bittinger, K.; et al. Association Between Breast Milk Bacterial Communities and Establishment and Development of the Infant Gut Microbiome. JAMA Pediatr. 2017, 171, 647-654, doi:10.1001/jamapediatrics.2017.0378.

34. Carlsson, J.; Grahnén, H.; Jonsson, G.; Wikner, S. Early Establishment of Streptococcus Salivarius in the Mouth of Infants. J. Dent. Res. 1970, 49, 415-418, doi:10.1177/00220345700490023601.

35. Cole, M.F.; Evans, M.; Fitzsimmons, S.; Johnson, J.; Pearce, C.; Sheridan, M.J.; Wientzen, R.; Bowden, G. Pioneer Oral Streptococci Produce Immunoglobulin A1 Protease. Infect. Immun. 1994, 62, 2165-2168, doi:10.1128/iai.62.6.2165-2168.1994.

36. Sampaio-Maia, B.; Monteiro-Silva, F. Acquisition and Maturation of Oral Microbiome throughout Childhood: An Update. Dent. Res. J. 2014, 11, 291-301.

37. Fernández, L.; Langa, S.; Martín, V.; Maldonado, A.; Jiménez, E.; Martín, R.; Rodríguez, J.M. The Human Milk Microbiota: Origin and Potential Roles in Health and Disease. Pharmacol. Res. 2013, 69, 1-10, doi:10.1016/j.phrs.2012.09.001.

38. Ruiz, L.; Bacigalupe, R.; García-Carral, C.; Boix-Amoros, A.; Argüello, H.; Silva, C.B.; de los Angeles Checa, M.; Mira, A.; Rodríguez, J.M. Microbiota of Human Precolostrum and Its Potential Role as a Source of Bacteria to the Infant Mouth. Sci. Rep. 2019, 9, 8435, doi:10.1038/s41598-019-42514-1.

39. Rajilić-Stojanović, M.; de Vos, W.M. The First 1000 Cultured Species of the Human Gastrointestinal Microbiota. FEMS Microbiol. Rev. 2014, 38, 996-1047, doi:10.1111/1574-6976.12075.

40. Gomez, A.; Nelson, K.E. The Oral Microbiome of Children: Development, Disease, and Implications Beyond Oral Health. Microb. Ecol. 2017, 73, 492-503, doi:10.1007/s00248-016-0854-1.

41. Sprockett, D.; Fukami, T.; Relman, D.A. Role of Priority Effects in the Early-Life Assembly of the Gut Microbiota. Nat. Rev. Gastroenterol. Hepatol. 2018, 15, 197-205, doi:10.1038/nrgastro.2017.173.

42. Cabrera-Rubio, R.; Collado, M.C.; Laitinen, K.; Salminen, S.; Isolauri, E.; Mira, A. The Human Milk Microbiome Changes over Lactation and Is Shaped by Maternal Weight and Mode of Delivery. Am. J. Clin. Nutr. 2012, 96, 544551, doi:10.3945/ajcn.112.037382.

43. Jost, T.; Lacroix, C.; Braegger, C.P.; Rochat, F.; Chassard, C. Vertical Mother-Neonate Transfer of Maternal Gut Bacteria via Breastfeeding. Environ. Microbiol. 2014, 16, 2891-2904, doi:10.1111/1462-2920.12238.

44. Crielaard, W.; Zaura, E.; Schuller, A.A.; Huse, S.M.; Montijn, R.C.; Keijser, B.J.F. Exploring the Oral Microbiota of Children at Various Developmental Stages of Their Dentition in the Relation to Their Oral Health. BMC Med. Genomics 2011, 4, 22, doi:10.1186/1755-8794-4-22.

45. Aas, J.A.; Paster, B.J.; Stokes, L.N.; Olsen, I.; Dewhirst, F.E. Defining the Normal Bacterial Flora of the Oral Cavity. J. Clin. Microbiol. 2005, 43, 5721-5732, doi:10.1128/JCM.43.11.5721-5732.2005.

46. Del Chierico, F.; Vernocchi, P.; Petrucca, A.; Paci, P.; Fuentes, S.; Praticò, G.; Capuani, G.; Masotti, A.; Reddel, S.; Russo, A.; et al. Phylogenetic and Metabolic Tracking of Gut Microbiota during Perinatal Development. PloS One 2015, 10, e0137347, doi:10.1371/journal.pone.0137347.

47. Jakobsson, H.E.; Abrahamsson, T.R.; Jenmalm, M.C.; Harris, K.; Quince, C.; Jernberg, C.; Björkstén, B.; Engstrand, L.; Andersson, A.F. Decreased Gut Microbiota Diversity, Delayed Bacteroidetes Colonisation and Reduced Th1 Responses in Infants Delivered by Caesarean Section. Gut 2014, 63, 559-566, doi:10.1136/gutjnl-2012-303249. 
48. Ardissone, A.N.; de la Cruz, D.M.; Davis-Richardson, A.G.; Rechcigl, K.T.; Li, N.; Drew, J.C.; Murgas-Torrazza, R.; Sharma, R.; Hudak, M.L.; Triplett, E.W.; et al. Meconium Microbiome Analysis Identifies Bacteria Correlated with Premature Birth. PloS One 2014, 9, e90784, doi:10.1371/journal.pone.0090784.

49. Matsuki, T.; Yahagi, K.; Mori, H.; Matsumoto, H.; Hara, T.; Tajima, S.; Ogawa, E.; Kodama, H.; Yamamoto, K.; Yamada, T.; et al. A Key Genetic Factor for Fucosyllactose Utilization Affects Infant Gut Microbiota Development. Nat. Commun. 2016, 7, 11939, doi:10.1038/ncomms11939.

50. Solís, G.; de Los Reyes-Gavilan, C.G.; Fernández, N.; Margolles, A.; Gueimonde, M. Establishment and Development of Lactic Acid Bacteria and Bifidobacteria Microbiota in Breast-Milk and the Infant Gut. Anaerobe 2010, 16, 307-310, doi:10.1016/j.anaerobe.2010.02.004.

51. Schwab, C.; Gänzle, M. Lactic Acid Bacteria Fermentation of Human Milk Oligosaccharide Components, Human Milk Oligosaccharides and Galactooligosaccharides: LAB Fermentation of HMOs and GOSs. FEMS Microbiol. Lett. 2011, 315, 141-148, doi:10.1111/j.1574-6968.2010.02185.x.

52. Zoetendal, E.G.; Raes, J.; van den Bogert, B.; Arumugam, M.; Booijink, C.C.G.M.; Troost, F.J.; Bork, P.; Wels, M.; de Vos, W.M.; Kleerebezem, M. The Human Small Intestinal Microbiota Is Driven by Rapid Uptake and Conversion of Simple Carbohydrates. ISME J. 2012, 6, 1415-1426, doi:10.1038/ismej.2011.212.

53. Human Microbiome Project Consortium Structure, Function and Diversity of the Healthy Human Microbiome. Nature 2012, 486, 207-214, doi:10.1038/nature11234.

54. Wang, M.; Li, M.; Wu, S.; Lebrilla, C.B.; Chapkin, R.S.; Ivanov, I.; Donovan, S.M. Fecal Microbiota Composition of Breast-Fed Infants Is Correlated with Human Milk Oligosaccharides Consumed. J. Pediatr. Gastroenterol. Nutr. 2015, 60, 825-833, doi:10.1097/MPG.0000000000000752.

55. Mueller, N.T.; Bakacs, E.; Combellick, J.; Grigoryan, Z.; Dominguez-Bello, M.G. The Infant Microbiome Development: Mom Matters. Trends Mol. Med. 2015, 21, 109-117, doi:10.1016/j.molmed.2014.12.002.

56. Murphy, K.; Curley, D.; O'Callaghan, T.F.; O'Shea, C.-A.; Dempsey, E.M.; O'Toole, P.W.; Ross, R.P.; Ryan, C.A.; Stanton, C. The Composition of Human Milk and Infant Faecal Microbiota Over the First Three Months of Life: A Pilot Study. Sci. Rep. 2017, 7, 40597, doi:10.1038/srep40597.

57. Lloyd-Price, J.; Mahurkar, A.; Rahnavard, G.; Crabtree, J.; Orvis, J.; Hall, A.B.; Brady, A.; Creasy, H.H.; McCracken, C.; Giglio, M.G.; et al. Strains, Functions and Dynamics in the Expanded Human Microbiome Project. Nature 2017, 550, 61-66, doi:10.1038/nature23889.

58. La Rosa, P.S.; Warner, B.B.; Zhou, Y.; Weinstock, G.M.; Sodergren, E.; Hall-Moore, C.M.; Stevens, H.J;; Bennett, W.E.; Shaikh, N.; Linneman, L.A.; et al. Patterned Progression of Bacterial Populations in the Premature Infant Gut. Proc. Natl. Acad. Sci. 2014, 111, 12522-12527, doi:10.1073/pnas.1409497111. 